\title{
Exploring the lengthiest ambulatory breast surgery clinic appointments: is the patient the problem?
}

\author{
Daniel J. Kagedan $^{1 \wedge}$, Kazuaki Takabe ${ }^{1,2}$ \\ ${ }^{1}$ Department of Surgical Oncology, Roswell Park Comprehensive Cancer Center, Buffalo, NY, USA; ${ }^{2}$ Jacobs School of Medicine and Biomedical \\ Sciences, University of Buffalo, Buffalo, NY, USA \\ Contributions: (I) Conception and design: All authors; (II) Administrative support: K Takabe; (III) Provision of study materials or patients: K Takabe; \\ (IV) Collection and assembly of data: DJ Kagedan; (V) Data analysis and interpretation: All authors; (VI) Manuscript writing: All authors; (VII) Final \\ approval of manuscript: All authors. \\ Correspondence to: Kazuaki Takabe, MD, PhD, FACS. Roswell Park Comprehensive Cancer Center, Clinical Sciences Center, Room P-615, Elm and \\ Carlton Streets, Buffalo, NY 14263, USA. Email: Kazuaki.Takabe@RoswellPark.org.
}

Background Prolonged outpatient clinic appointments can disrupt schedules, impacting patients and providers. We sought to define features of the longest ambulatory appointments in a breast surgery clinic, and to ascertain whether patients attending these appointments consistently have protracted appointments.

Methods: A single-institution retrospective cohort study was conducted of breast surgery clinic patients, January 2017 to May 2019, and the longest 1\% of appointments identified using a real-time patient tracking system. A primary chart review was performed, and data abstracted. Other appointments attended by these patients were identified, and the ratio of appointments $>75$ th percentile duration (protracted appointments) to the total appointments during the study period was calculated, enabling comparison for patients with consistently protracted (ratio $>50 \%$ ) vs. sporadically protracted appointments $(\leq 50 \%)$. Descriptive analysis was performed, and results reported as medians with inter-quartile ranges.

Results: A total of 15,265 clinic appointments were identified, and the longest 148 (exceeding 244 minutes) analyzed. Median appointment length was 264 minutes (inter-quartile range: $253-290$ ). $70 \%$ were new patient appointments, and $54 \%$ of patients underwent a test/investigation that day. A minority were obese (39\%), smokers (41\%), diagnosed with a psychiatric comorbidity (34\%), had a genetic cancer syndrome $(22 \%)$, or received unexpected news at their appointment (16\%). Of 118 patients with multiple appointments, 26\% had consistently protracted appointments and $74 \%$ sporadically protracted appointments.

Conclusions: The lengthiest appointments are usually newly diagnosed cancer patients. Only a minority of patients have consistently protracted appointments, implying that a patient's previously prolonged appointment may not predict future long appointments.

Keywords: Breast surgery; breast cancer; appointment length; clinic visits; time factors

Submitted Jul 22, 2020. Accepted for publication Nov 21, 2020.

doi: 10.21037 /gs-20-623

View this article at: http://dx.doi.org/10.21037/gs-20-623

^ ORCID: 0000-0002-4200-198X. 


\section{Introduction}

Outpatient clinic appointments are ubiquitous in clinical medicine and surgery. Myriad factors pressure physicians and healthcare providers to spend only as much time as is needed on each encounter (1). Several variables can increase or decrease ambulatory visit duration including disease complexity, the involvement of trainees, and patient factors such as late arrival time (2-4); however, there is a paucity of data regarding the extreme outliers whose appointments are the longest. Lengthy appointments can disrupt schedules for providers, patients, and families, both those directly involved in the protracted appointment, and everyone seen hence in clinic that day (5). This can in turn affect patient satisfaction and the perception of care received, with ramifications for institutional funding and referrals (6). It is not known whether the individual patient is the primary determinant of an extremely long appointment, or if other factors such as unexpected findings to communicate or a large number of pre-appointment tests to review are the principal contributors.

We sought to elucidate the longest appointments and to describe the patient- and appointment-related factors underlying them in an ambulatory breast surgery clinic setting at a high-volume cancer center. We attempted to ascertain the degree to which those patients attending the longest appointments consistently had appointments of protracted duration, or whether these lengthy appointments were unrelated to the patients themselves. We hypothesized that those patients attending the longest appointments would consistently have lengthy appointments. To the authors' knowledge, this is the first study investigating whether those patients with lengthy clinic appointments have consistently versus sporadically protracted appointments. We present the following article in accordance with the STROBE reporting checklist (available at http://dx.doi.org/10.21037/gs-20-623).

\section{Methods}

\section{Study cobort and database description}

The study was designed as a single-institution retrospective cohort study. Outpatient breast surgery clinic appointments at a single institution (Roswell Park Comprehensive Cancer Center) from January 1, 2017 to May 21, 2019 (inclusive) were identified, and the corresponding data from a realtime patient tracking system included in this study. Each appointment and the medical record number (MRN) corresponding to the patient attending that appointment was captured in the dataset. The appointment duration, measured from check in time to check out time [total appointment length (TAL)], was calculated using the precise clock times recorded by the clinic registration staff, rounded to the nearest minute. Appointments missing values for either check in or check out times were excluded from analysis. Time spent away from the breast surgery clinic (i.e., if a patient was seen in the clinic, then was sent for an imaging test, then returned to clinic with the results) was subtracted from the appointment duration from the time the patient left the clinic area to the time they returned. The patient tracking system also recorded the clinicians involved in each appointment, including trainees (residents or fellows) and advanced practice providers (APPs). Trainees saw patients under the attending surgeon's supervision (the trainee saw the patient first, then the attending). APPs saw some patients under the attending surgeon's supervision, and other patients independently for pre-determined designated appointments (i.e., surveillance, high risk follow-up).

Appointments were categorized into six types based on patient history (for established patients) and referral information (for new patients): new malignant (new patient diagnosed with cancer); new benign (new patient with benign disease); new high risk (new patient for high risk evaluation); pre-operative (established patient seen prior to operation); post-operative (established patient seen at first appointment after operation); and follow-up (established patient seen for surveillance, screening, monitoring). The classification of appointment type was compiled prior to the clinic in a daily appointment list, which was linked to the appointment duration dataset.

\section{Patient and appointment variables}

Appointment duration was treated as a continuous variable. The longest $1 \%$ of appointments were identified, and a primary chart review performed of corresponding clinic records (notes) contained in the institutional electronic medical record. Data extracted included AM vs. PM appointment start time, appointment type, the type of clinicians involved (attending MDs, APPs, trainees), the scheduled appointment duration (30, 45, or 60 minutes), and whether the patient received unexpected news at that appointment. Unexpected news was defined as including a diagnosis of recurrent cancer, as well as other findings identified by either the healthcare provider or patient such as positive genetic test results. Scheduled 
appointment duration was determined prior to the visit by the clinic scheduling team, and generally corresponded to the appointment type (new patients scheduled for 60 minutes, routine follow-up appointments for 30 minutes). Also recorded were the number of test results (imaging studies, pathology reports, genetic tests) reviewed at that appointment, calculated by counting the number of test results included in that clinic note, as well as whether or not the patient underwent a test (imaging, biopsy) at the institution that day. Routine laboratory tests (i.e., bloodwork) were not included. The patient corresponding to each appointment was identified using their MRN, and the following data abstracted for each patient: age at appointment; body mass index (BMI), number of comorbidities (excluding breast disease); presence of one or more psychiatric comorbidities; smoking status (current or former smoker $v s$. never smoker); and presence of a known or suspected genetic disorder.

\section{Consistently protracted vs. sporadically protracted appointments}

The 75th percentile appointment duration was calculated for each appointment type using all of the appointments attended by all patients at the breast surgery clinic during the study period. Appointment durations exceeding the 75 th percentile (upper quartile) for the corresponding appointment type were termed "protracted appointments". Using the MRNs of patients with the longest $1 \%$ of appointments, all other breast surgery clinic appointments attended by these patients during the study period were identified and the duration of each appointment calculated. Thus, the number of protracted appointments was determined for each patient with one or more appointments in the top $1 \%$. For each of these patients, the ratio of protracted appointments to total appointments during the study period was calculated, and the number of patients with a ratio greater than $50 \%$ was determined. These patients were deemed as having consistently protracted appointments, versus the others who had sporadically protracted appointments. Patients with only a single appointment during the study period were not categorized as having either consistently or sporadically protracted appointments.

\section{Statistical analyses}

Descriptive analysis was performed and non-parametric tests (Mann-Whitney U, Kruskal-Wallis) used to compare patient characteristics between individuals with consistently protracted $v s$. sporadically protracted appointments. Results were presented as median values with inter-quartile ranges (IQRs). Due to the small size of the cohort, multivariable analysis was not performed. Two-sided hypothesis testing was employed for statistical analyses, and statistical significance established at an alpha of 0.05 . SPSS version 25 was used for all analyses.

\section{Ethics statement}

The study was conducted in accordance with the Declaration of Helsinki (as revised in 2013). The study was approved by the institutional ethics board of Roswell Park Comprehensive Cancer Center (No. 00001124) and individual consent for this retrospective analysis was waived.

\section{Results}

\section{Cohort description}

There were 15,265 appointments in the ambulatory breast surgery clinic between January 1, 2017 and May 23, 2019. Of these, appointments missing a valid check out (clinic departure) time $(\mathrm{N}=1,102)$ and those missing a valid check in (clinic arrival) time $(\mathrm{N}=135)$ were excluded. The longest $1 \%$ of TALs among the remaining 14,028 appointments included all appointments equal to or exceeding 244.0 minutes in duration. There were 148 appointments in the longest $1 \%$ subgroup, with a median duration of 264.0 minutes (IQR: 253.0-289.75). The longest appointment included lasted 438.0 minutes. The clinician and appointment types of the longest 1\% subgroup are presented in Figure 1. Most appointments (69.6\%) were new patients (56.8\% malignant, $12.8 \%$ benign), and $70.3 \%$ were seen by an APP plus attending MD.

\section{Appointment characteristics}

The characteristics of the longest $1 \%$ of appointments are presented in Figure 2. Approximately half (54.1\%) occurred on the same day as the patient underwent one or more tests (imaging study and/or percutaneous procedure) at the institution. A minority of the longest appointments included the delivery of unexpected news or diagnosis of recurrence. Notably, 40 of the longest appointments were booked for 30 minutes duration (the shortest amount of time). The median number of tests reviewed (including imaging, biopsies, and genetics) in the longest $1 \%$ of appointments 
A

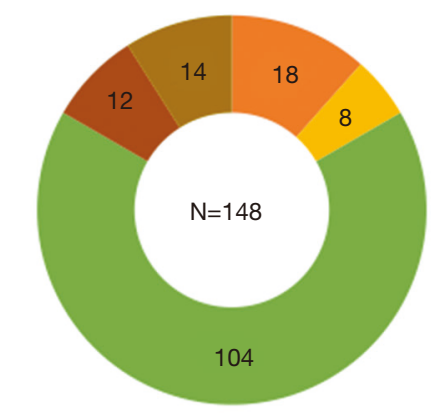

$=$ Attending alone $\quad$ APP alone
$=$ APP + attending $\quad$ Fellow + attending
$=$ Resident + attending
B

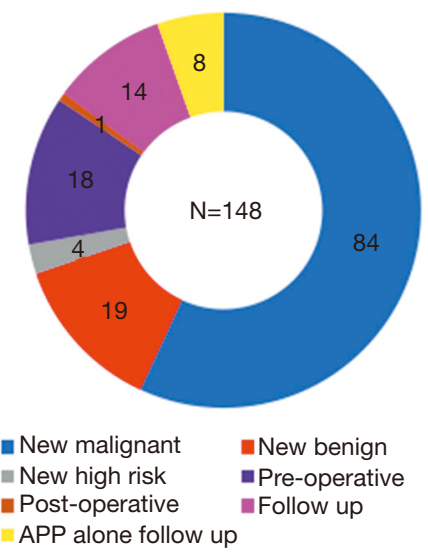

Figure 1 Number of the lengthiest 1\% of breast clinic patient appointments (148 total) subdivided by: (A) clinician type(s) involved in each appointment and (B) appointment type. APP, advanced practice provider.
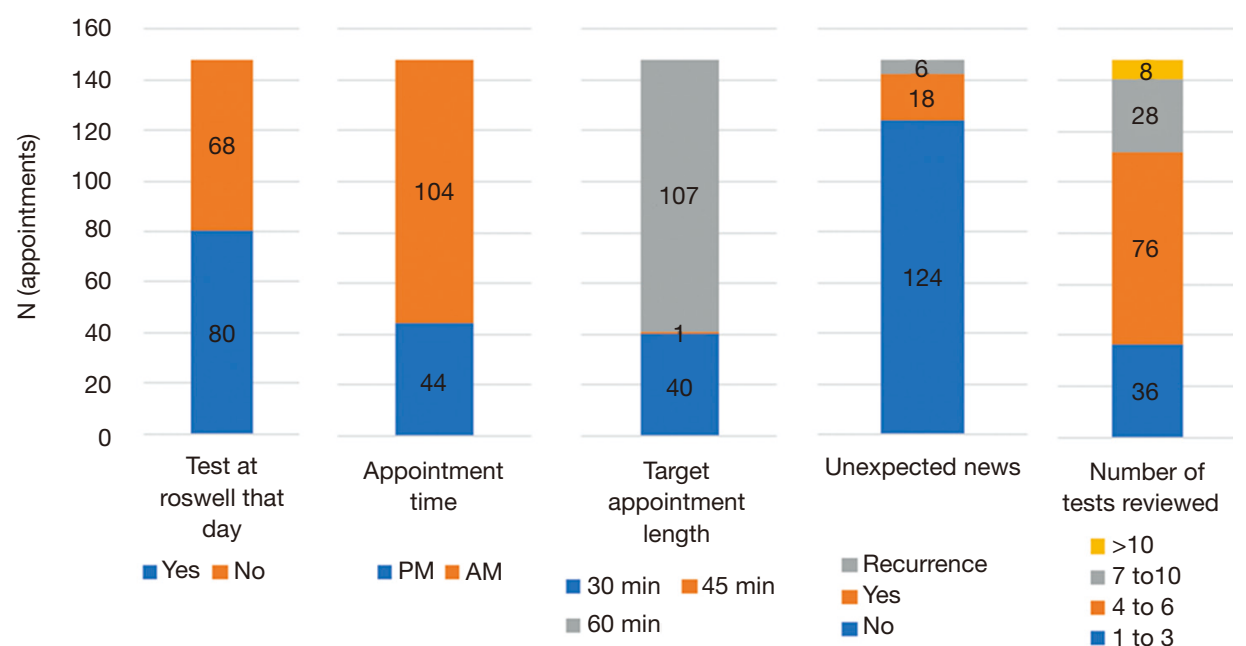

Figure 2 Description of longest 148 appointments (lengthiest 1\%) in terms of appointment-level factors/variables.

was 5 (IQR: $1-17$ ), and $75 \%$ had 6 or fewer tests reviewed in their clinic note. There were no recorded instances of clinic procedures (i.e., skin biopsy, seroma aspiration) performed during any of these appointments.

\section{Patient characteristics}

Attending the 148 longest appointments were 145 individual patients, with 3 patients each having 2 appointments, and 142 patients having 1 appointment. The characteristics of the included patient cohort is presented in Figure 3. All patients were female, and the median age was
59.0 years old (IQR: 50-67). A minority of patients were obese $(38.9 \%)$, current or former smokers $(40.7 \%)$, diagnosed with a psychiatric comorbidity $(33.8 \%)$, or had a known or suspected genetic cancer syndrome (22.1\%).

\section{Consistently protracted vs. sporadically protracted appointments}

Among the 145 patients with the longest appointments, 27 had only a single appointment recorded during the study period (i.e., the longest appointment), and 118 patients had multiple appointments documented (i.e., one or more 

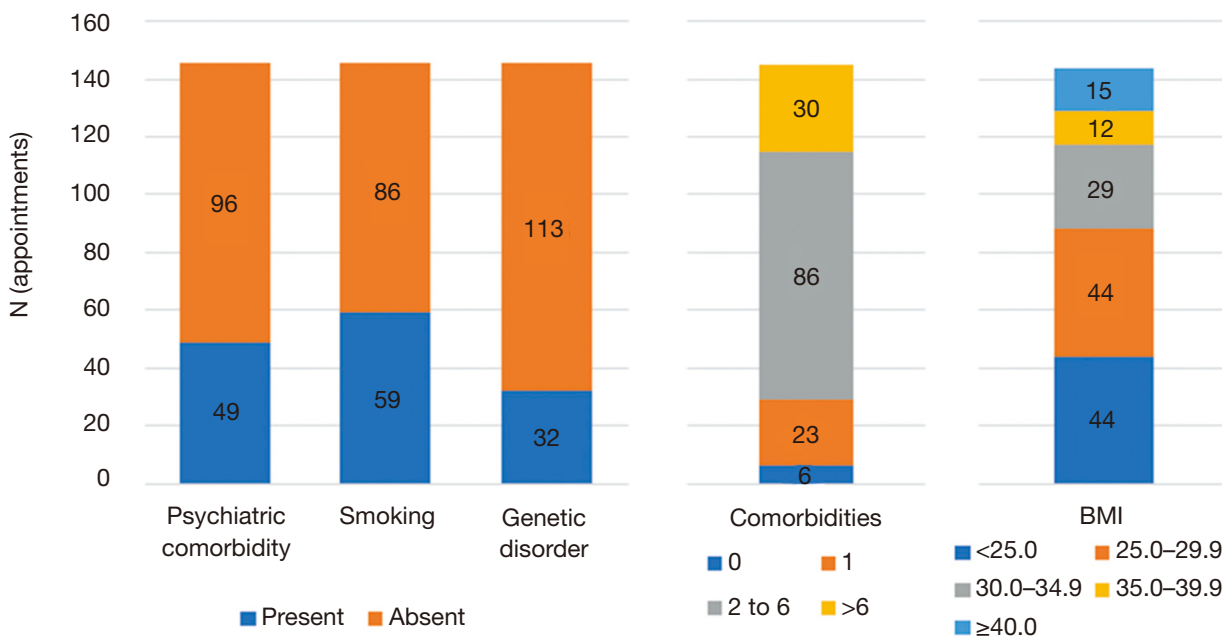

Figure 3 Description of patients $(\mathrm{N}=145)$ attending the lengthiest $1 \%$ of appointments in terms of patient-level factors/variables.

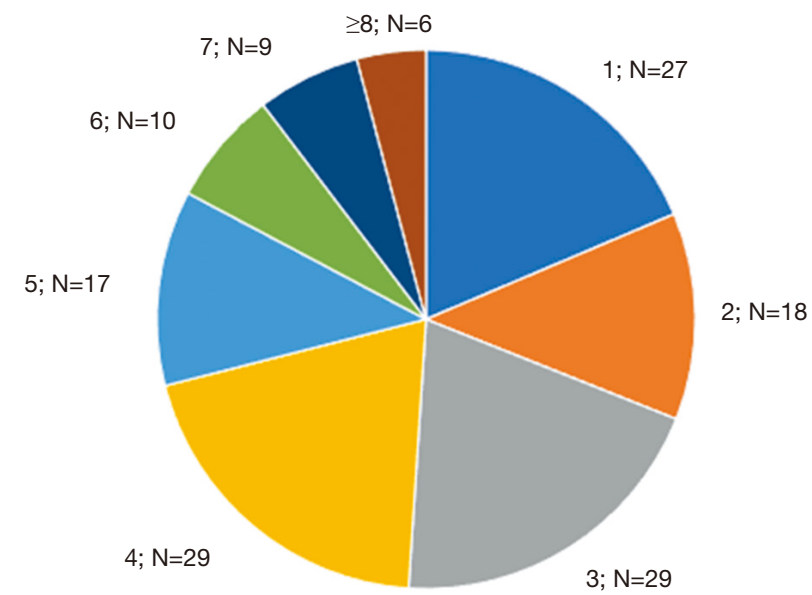

Figure 4 Total number of appointments attended by each patient during the study period who had at least one appointment in the lengthiest $1 \%$.

appointments in addition to the longest appointment). The number of appointments during the study period per patient is displayed in Figure 4.

Of the 118 patients with multiple appointments, 31 patients $(26.3 \%)$ had $>50 \%$ of their appointments exceed the 75 th percentile of time length for that appointment type (i.e., consistently had protracted appointments), and $87(73.7 \%)$ had $\leq 50 \%$ in the top quartile of time lengths (i.e., sporadically had protracted appointments) (Figure 5). Patients from these two groups were similar with respect to age $(\mathrm{P}=0.273)$, comorbidity burden $(\mathrm{P}=0.534)$, psychiatric comorbidity $(\mathrm{P}=0.745)$, smoking status $(\mathrm{P}=0.151)$, BMI
$(\mathrm{P}=0.629)$, and presence or suspicion of a genetic cancer syndrome $(\mathrm{P}=0.825)$.

\section{Discussion}

This study describes characteristics of the lengthiest outpatient breast surgery clinic appointments at an academic cancer center, and the patients attending these appointments. It reports that only a minority of these patients had appointments that were consistently protracted in duration. Moreover, among patients with the longest appointments, those with consistently protracted appointments were similar to those whose appointments were most often of shorter duration (sporadically protracted appointments) in terms of the patient-related characteristics investigated. The decision to limit the cohort of appointments investigated to those at the breast surgery clinic was based on the observation that the time stamps were less reliably recorded in the patient tracking system in other clinics.

New patient visits comprised the majority of the longest appointments. This association has previously been reported in the literature in other specialties (7). Shorter duration of preoperative and postoperative appointments among cancer patients has also been reported (8). This suggests that the initial assessment and formulation of a management plan contributes more to the longest appointment duration than interpretation and discussion of postoperative results, and communication of unexpected findings on surveillance, including cancer recurrence. This may relate to an emphasis 


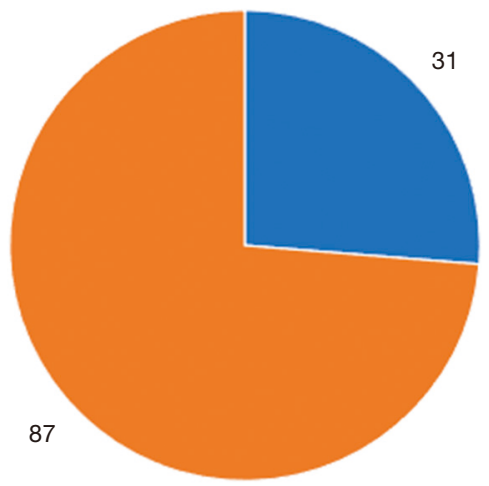

n $>50 \%$ above top quartile $\leq 50 \%$ above top quartile

Figure 5 Number of patients (all with at least one appointment in the lengthiest $1 \%$ ) with multiple appointments during the study period $(\mathrm{N}=118)$, consistently protracted appointments ( $>50 \%$ above the top quartile, $\mathrm{N}=31$ ), and sporadically protracted appointments ( $\leq 50 \%$ above the top quartile, $\mathrm{N}=87$ ).

on comprehensive explanation and answering of current and anticipated future questions at the initial appointment. Number of diagnostic tests has also been reported to be independent of visit duration (8).

This study reports that only a minority of patients with the longest appointments were obese, diagnosed with a pre-existing mental illness, current/former smokers, or known/suspected to have a familial cancer syndrome. The proportion of patients with each of these characteristics in the longest appointments cohort was similar to the reported prevalence of smoking history (50\%) (9), pre-existing mental illness (25\%) (10), obesity (11), and known or suspected familial/genetic cancer syndromes (20\%) (12) among all breast cancer patients. Moreover, obese patients have previously been shown not to have statistically significantly longer appointments (13). Similar appointment duration across patient age groups has also been reported (14). This implies that none of these factors predict extremely long appointments. However, given that we did not compare these variables with their frequency in the larger cohort (i.e., the $99 \%$ with shorter visit length), these findings should be interpreted with caution.

Patients seen only once during the study period comprised $18.6 \%$ of the cohort. The majority of these single appointments presumably represent patients who chose to seek their treatment/follow up elsewhere (i.e., sought a second opinion). This rate is approximately equal to that reported in the general population in an Israeli cohort of patients (15), implying that these patients do not more frequently take an exceedingly long time during their appointment.

The data suggest that of those patients with the longest appointments in breast surgery clinic, only a minority consistently have appointments protracted in duration. For most, the exceedingly lengthy appointment is not the norm. This finding is somewhat surprising, and implies that patient and disease factors may not be the principal determinants of exceedingly long appointments in this population. Therefore, it may be clinic and provider factors that play a more significant role in breast surgery clinic, and this warrants further investigation.

There are several limitations to this study, including the retrospective design and the utilization of a time-stamp tracking system that depends on manual entry of data. However, a study from a different institution found that time stamp patient tracking systems were highly concordant with actual patient flow during a clinic appointment (within 3 minutes for $>80 \%$ of appointments) (16). Another important limitation is the lack of patient- and appointmentspecific data for the larger cohort of patients/appointments (i.e., the $99 \%$ with shorter appointment duration). This is a result of the databases interrogated and the information available for analysis, which required a primary chart review to extract this data, and was not feasible for the entire cohort of 14,028 appointments. Accordingly, this section of the results should be interpreted with caution, and the patient- and appointment-related variables reported should be viewed as a descriptive analysis of the lengthiest $1 \%$ of appointments, as opposed to concluding an association (or lack thereof) between any of these factors and extreme appointment duration. Lastly, the findings are derived from a cohort of patients seen in a breast surgery clinic at a dedicated cancer center, and caution should be exercised in generalizing them to other populations. The degree to which they are applicable to patients with similar disease processes seen in other institutions or other regions is uncertain. As this study reports on patient interactions with healthcare providers, which are highly influenced by cultural and sociodemographic variables, they may differ substantially in other countries, and further study of this subject using similar methods in other contexts is warranted.

\section{Conclusions}

In conclusion, the majority of the lengthiest $1 \%$ of appointments in the ambulatory breast surgery clinic 
studied were for newly diagnosed breast malignancy. Only a minority $(26 \%)$ of patients had consistently protracted appointments. This has implications for clinic scheduling, in that a patient's previously extremely lengthy appointment may not predict future long appointments. These results are limited in external validity/generalizability, as sociodemographic, clinicopathologic, healthcare system and cultural factors all contribute to the outcomes investigated, and should be investigated in other contexts before being broadly applied. Further investigation into the association of specific patient, provider, and clinic factors with appointment duration is needed, ideally using a prospective study design or techniques to account for the limitations inherent to retrospective study designs.

\section{Acknowledgments}

The authors would like to acknowledge the assistance of Kevin Davenport and his team in maintaining the patient tracking system database at Roswell Park Comprehensive Cancer Center, and providing the breast clinic data contained therein for this project.

Funding: This work was supported by a National Institute of Health grant (R01CA 160688) to KT.

\section{Footnote}

Reporting Checklist: Both authors have completed the STROBE reporting checklist. Available at http://dx.doi. org/10.21037/gs-20-623

Data Sharing Statement: Available at http://dx.doi. org/10.21037/gs-20-623

Conflicts of Interest: Both authors have completed the ICMJE uniform disclosure form (available at http://dx.doi. org/10.21037/gs-20-623). The authors have no conflicts of interest to declare.

Ethical Statement: The authors are accountable for all aspects of the work in ensuring that questions related to the accuracy or integrity of any part of the work are appropriately investigated and resolved. The study was conducted in accordance with the Declaration of Helsinki (as revised in 2013). The study was approved by the institutional ethics board of Roswell Park Comprehensive Cancer Center (No. 00001124) and individual consent for this retrospective analysis was waived.
Open Access Statement: This is an Open Access article distributed in accordance with the Creative Commons Attribution-NonCommercial-NoDerivs 4.0 International License (CC BY-NC-ND 4.0), which permits the noncommercial replication and distribution of the article with the strict proviso that no changes or edits are made and the original work is properly cited (including links to both the formal publication through the relevant DOI and the license). See: https://creativecommons.org/licenses/by-nc-nd/4.0/.

\section{References}

1. Hu P, Reuben DB. Effects of managed care on the length of time that elderly patients spend with physicians during ambulatory visits: National Ambulatory Medical Care Survey. Med Care 2002;40:606-13.

2. Goldstein IH, Hribar MR, Read-Brown S, et al. Association of the Presence of Trainees With Outpatient Appointment Times in an Ophthalmology Clinic. JAMA Ophthalmol 2018;136:20-6.

3. Medway AM, de Riese WT, de Riese CS, et al. Why patients should arrive late: The impact of arrival time on patient satisfaction in an academic clinic. Healthc (Amst) 2016;4:188-91.

4. Zhu Z, Heng BH, Teow KL. Analysis of factors causing long patient waiting time and clinic overtime in outpatient clinics. J Med Syst 2012;36:707-13.

5. Chung S, Johns N, Zhao B, et al. Clocks Moving at Different Speeds: Cultural Variation in the Satisfaction With Wait Time for Outpatient Care. Med Care 2016;54:269-76.

6. Bleustein C, Rothschild DB, Valen A, et al. Wait times, patient satisfaction scores, and the perception of care. Am J Manag Care 2014;20:393-400.

7. Ananthakrishnan AN, McGinley EL, Saeian K. Length of office visits for gastrointestinal disease: impact of physician specialty. Am J Gastroenterol 2010;105:1719-25.

8. Guy GP Jr, Richardson LC. Visit duration for outpatient physician office visits among patients with cancer. J Oncol Pract 2012;8:2s-8s.

9. Passarelli MN, Newcomb PA, Hampton JM, et al. Cigarette Smoking Before and After Breast Cancer Diagnosis: Mortality From Breast Cancer and SmokingRelated Diseases. J Clin Oncol 2016;34:1315-22.

10. Haskins CB, McDowell BD, Carnahan RM, et al. Impact of preexisting mental illness on breast cancer endocrine therapy adherence. Breast Cancer Res Treat 2019;174:197-208. 
11. Engmann NJ, Golmakani MK, Miglioretti DL, et al. Population-Attributable Risk Proportion of Clinical Risk Factors for Breast Cancer. JAMA Oncol 2017;3:1228-36.

12. Jacobi CE, Jonker MA, Nagelkerke NJ, et al. Prevalence of family histories of breast cancer in the general population and the incidence of related seeking of health care. J Med Genet 2003;40:e83.

13. Pearson WS, Bhat-Schelbert K, Ford ES, et al. The impact of obesity on time spent with the provider and number of medications managed during office-based physician visits using a cross-sectional, national health survey. BMC Public

Cite this article as: Kagedan DJ, Takabe K. Exploring the lengthiest ambulatory breast surgery clinic appointments: is the patient the problem? Gland Surg 2021;10(2):551-558. doi: 10.21037 /gs-20-623
Health 2009;9:436.

14. Lo A, Ryder K, Shorr RI. Relationship between patient age and duration of physician visit in ambulatory setting: does one size fit all? J Am Geriatr Soc 2005;53:1162-7.

15. Shmueli L, Shmueli E, Pliskin JS, et al. Second Medical Opinion: Utilization Rates and Characteristics of Seekers in a General Population. Med Care 2016;54:921-8.

16. Hribar MR, Read-Brown S, Goldstein IH, et al. Secondary use of electronic health record data for clinical workflow analysis. J Am Med Inform Assoc 2018;25:40-6. 